\title{
Infrastructure for Innovative Research on Healthy Food Choice, Preparation and Consumption: A Position Paper on the RICHFIELDS project
}

\author{
Barbara Koroušić Seljak \\ Computer Systems \\ Jožef Stefan Institute \\ Ljubljana, Slovenia \\ barbara.korousic@ijs.si
}

Lada Timotijević

Food, Consumer Behaviour and Health

Research Centre, School of Psychology

University of Surrey

Guildford, Surrey

1.timotijevic@surrey.ac.uk

\author{
Krijn Popp \\ Wageningen Economic Research \\ The Haag, Netherlands \\ krijn.poppe@wur.nl \\ Pieter van't Vee \\ Wageningen Economic Research
}

The Haag, Netherlands

pieter.vantveer@wur.nl

\author{
Paul Finglas \\ Quadram Institute Bioscience \\ Norwich, UK \\ paul.finglas@quadram.ac.uk \\ Karin Zimmerman \\ Wageningen Economic Research \\ The Haag, Netherlands \\ karin.zimmermann@wur.nl
}

\begin{abstract}
This paper presents the recently finished EUfunded RICHFIELDS project aimed to design a new research infrastructure that would foster research in the areas of food and nutrition with a focus on consumers' behavior and lifestyle. In this project, an architecture of a new consumer data platform was designed and discussed from the researchers, business, management, ethical and legal points of view. Also new methodology for supporting big and open data standardization and interoperability was developed.
\end{abstract}

Keywords-data, food choice, nutrition, consumer, interoperability

\section{INTRODUCTION}

RICHFIELDS is the acronym of »Research Infrastructure on Consumer Health and Food Intake using E-science with Linked Data Sharing«. The RICHFIELDS project was submitted in August 2014 to the Directorate General for Research and Innovation of the European Commission, following a call of the framework Excellent Science to tender on »Research infrastructers design studies«. The RICHFIELDS project received top marks in the evaluation procedure, and was performed in the period from the automn 2015 until the summer 2018. The aim of this paper is to present the key characteristics of the RICHFIELDS project, highlighting in particular the cornerstones of its approach to the design study of research infrastructures today.

In Section II, we begin by presenting the call to tender issued by the European Commission and characterise RICHFIELDS's response to this call, highlighting in particular two interrelated features: first, its focus on the questions raised by the call; second, its commitment to interdisciplinary work. In Section III, we present the project's core approach and its structural design.

As a conclusion, Section IV provides a discussion on the main outcomes of the project.

Let us draw the reader's attention to the fact that this paper is not about the development of the data platform itself, but about how, in a scientific context, its design was prepared through a large-scale research project. Accordingly, the scientific substance of the project is only addressed in general terms as the RICHFIELDS outcomes are presented in details in the project's deliverables [1].

\section{THE DESIGN OF THE RICHFIELDS PROJECT}

\section{A. The Call to Tender}

The European Commission's call to tender for a project on "Design studies" (H2020-INFRADEV-1-2014-1) presented two noteworthy features: first, it posed a wide range of questions about principle features and feasibility: »Design studies should tackle all the key questions concerning the technical and conceptual feasibility of new or upgraded fully fledged user facilities and a proposal should demonstrate the relevance and the advancement with respect to the state-of-art of the proposed infrastructure«. Second, it requested an interdisciplinary approach, by explicitly naming transdisciplinarian academic areas. Currently, there are no international research infrastructures to connect diverse data and science-related services in the field of food, consumer choice, behaviour and health (nutrition) that would aid science in this domain [2]. The RICHFIELDS successfully addressed both aspects focussing on detailed description of the context and environments in which the food-related consumer behaviour is taking place, purchase, preparation and consumption.

The range of questions to be addressed included:

- Could RICHFIELDS design a world class research infrastructure (RI) on Food and Health Consumer Behaviour and Lifestyle, that will serve as an open access, distributed data-platform to collate and collect, align and share innovative and existing data?

- Could RICHFIELDS design a RI to support integration of large diversity of data that describes the interactions of time, place and individuals' behaviour? And can this be achieved by integrating food- and nutrition-related data generated either for the purposes of research or generated for the purposes other than research (e.g. commerce); generated either directly by consumers or indirectly by technology (e.g. apps, sensors) or information systems/processes (e.g. medical data, sales data, surveillance data)?

- Could RICHFIELDS design a platform for foodrelated consumer behaviour and lifestyle that will enable scientifically sound inference by connecting data and methods from correlational sources (open, 
web-based, unstructured) with established observational (epidemiology, surveillance, purchase) and experimental data/methods (experimental kitchen, buffet, restaurant)?

- Could RICHFIELDS design a platform that fosters the interaction with new technologies and bring food and health research and dietary surveillance to the next level, in order to attract young researchers and build new research communities?

\section{B. Meeting the Call's Demands}

Preparations for the RICHFIELDS project started with an in-depth examination of the questions addressed by the call. In order to answer these questions, the RICHFIELDS core consortium adopted three principles: (1) interdisciplinarity, (2) a focus on the development of advanced methododology, and (3) involvement of different stakeholders.

To overcome the challenge of interdisciplinarity, scientists from the fields of food and nutrition, epidemiology, genomics, psychology, computer science, business and marketing, and law were involved.

As data science from the perspective of a consumer, which was the core of the project RICHFIELDS, is still under development, new advanced methodology for food- and nutrition-related data collection, harmonisation and analysis was suggested to be developed in the project.

In the project, there were involved stakeholders such as policy makers, industry and consumer groups.

\section{The Performance of the RICHFIELDS ProJeCT}

The project acronym highlights »research«, »innovation« and »food choice $«$. By »food choice $«$. we mean the fact that a consumer is confronted with a trade-off between biological, psychological, economic, sociocultural and environmental aspects of food and nutrition. In order to better understand this trade-off, scientific questions need to be tackled considering diverse data.

In the RICHFIELDS project, the work was performed in three main Phases: (1) "Data generated by consumers on", (2) "Connecting business and research generated data", and (3) "Design of the research infrastructure".

\section{A. Phase 1 and Phase 2}

In first two phases, the data collection and analysis processes were carried out. In Phase 1, focus was given on big data generated by consumers during food purchase, preparation and consumption, while in Phase 2, business and research-generated data were discussed. Using scientific methodology, potential sources of both big and open data that follow the FAIR Guiding Design principles [3] were identified and explored. Determinants of dietary behavior, food consumption and nutrition, including food components, were identified [11].

It was assessed how people generating data are willing to share their data for research and business purposes. In the RICHFIELDS deliverables [1], the results of this assessment have been presented.

We also identified and assessed the needs and perspectives of the potential users (i.e. professors, researchers, doctoral candidates, technical staff, and students) of food- and nutrition-related data addressed by the RICHFIELDS project.

\section{B. Phase 3}

In Phase 3, first, needs for a new research infrastructure (RI) for food- and nutrition-related data related to consumers as data generators and researchers as data users were identified and formalised. Then, (i) an architecture of the RICHFIELDS consumer data platform was designed but not implemented, (ii) its business model was developed, and (iii) organisational governance considering legal and ethical aspects was discussed. The overall methodogical approach taken in this phase, is presented in details in [4].

Regarding the architecture of the RICHFIELDS consumer data platform, we decided for a distributed system connecting different-data providing systems or data-processing systems, where data providers prepare data for themselves and expose it to the platform and its connected systems via web services. In order to minimize potential risks to personal data, we designed technical solutions based the processes of pseudonymisation, as part of data architecture.

In Fig. 1, the architecture of the RICHFIELDS consumer data platform (RDP) is presented. For each connected system providing data, the RDP will automatically collect metadata needed to create knowledge [5], which is required for data standardisation and interoperability. In this way, we will be able to handle with different types of data (such as structured, semi-structured and unstructured data), being described and classified using different systems. The result of this knowledge extraction will be a new ontology (in Fig. 1 named the RICHFIELDS ontology) that will be linked with other food- and nutrition-related ontologies.

In the project RICHFIELDS, for example, we developed advanced methods for food image recognition, which enables structuring of food information from photos [6] and linking it with already structured data, for example, with food composition data [7]. Other examples of structuring data include (i) extraction of dietary recommendations from scientific papers or reports published online $[8,9]$ and (ii) matching food composition data to food consumption data, where both systems are described using different systems, such as LanguaL and FoodEx2 [10].

The module, in Fig. 1 named "RICHFIELDS information system managing knowledge", is aimed to store and manage the knowledge extracted from data. This will be the most important module running services for data linkage and harmonisation. The central part will serve as an API management system connecting information systems providing data with systems requesting data.

\section{DISCUSSION}

In Europe, there is still a lack of well-established RIs specific to food and nutrition. The ambition of the RICHFIELDS project was to design such a RI and contribute to the development of interdisciplinary research of foodrelated consumer behavior and lifestyle.

In the area of data science, the main contribution of the RICHFIELDS project is a methodology for the standardisation and interoperability of food- and nutritionrelated data.

The prepared design and methodology is ready to follow up as a building block of a larger Food, Nutrition and Health Research Infrastructure (FNH-RI) that is currently under preparation. 


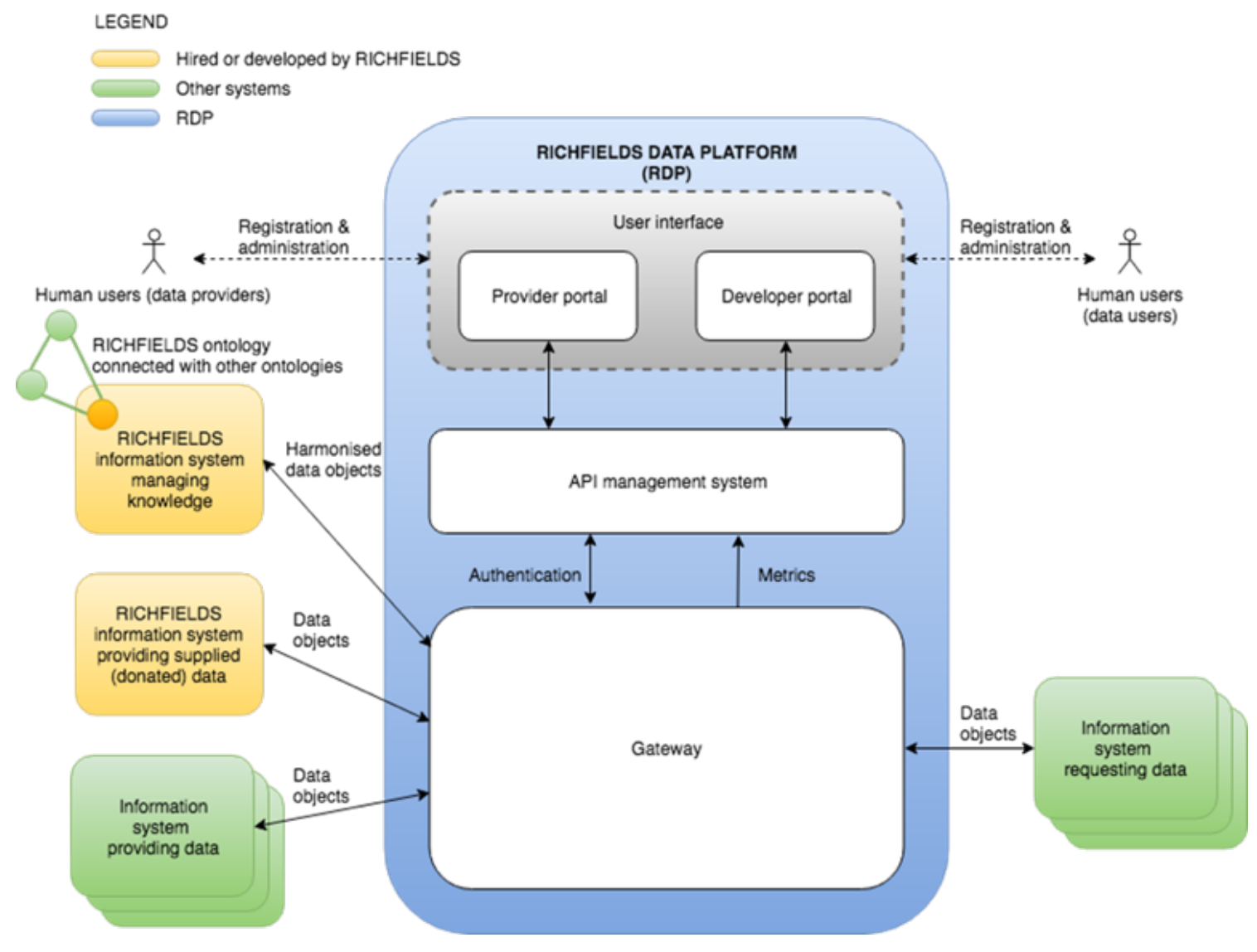

Fig. 1. The architecture of the RICHFIELDS Data Platform.

\section{Discussion}

In Europe, there is still a lack of well-established RIs specific to food and nutrition. The ambition of the RICHFIELDS project was to design such a RI and contribute to the development of interdisciplinary research of foodrelated consumer behavior and lifestyle. In the area of data science, the main contribution of the RICHFIELDS project is a methodology for the standardisation and interoperability of food- and nutrition-related data. The prepared design and methodology is ready to follow up as a building block of a larger Food, Nutrition and Health Research Infrastructure (FNH-RI) that is currently under preparation.

\section{ACKNOWLEDGMENT}

This project received funding from the European Union's Horizon 2020 programmes $(654280,863059,769661)$ and from the Slovenian Research Agency (No. P2-0098).

\section{REFERENCES}

[1] The project RICHFIELDS web site. Available at: https://www.richfields.eu (accessed on $3^{\text {rd }}$ October 2019).

[2] K.A. Brown, L. Timotijević, M. Geurts, J.L. Arentoft, R.A.M. Dhonukshe-Rutten, L. Fezeu, L., ... K.L. Zimmermann. "Concepts and procedures for mapping food and health research infrastructure: New insights from the EuroDISH project", Trends in Food Science and Technology, 2017, 63:113-131. doi:10.1016/j.tifs.2017.03.006.

[3] M. Wilkinson, M. Dumontier, I, J. Aalbersberg, G. Appleton, M. Axton et al. B. Mons, "The FAIR Guiding Principles for scientific data management and stewardship", Scientific Data, 3, 2016, 10.1038/sdata.2016.18.
[4] M.-J. Bogaardt, A. Geelen, K. Zimmermann, P. Finglas, M. M. Raats, et al, and P. van't Veer, "Designing a research infrastructure on dietary intake and its determinants", Nutrition Bulletin, 43, pp. 301-309, 2018, doi: $10.1111 /$ nbu. 12342 .

[5] T. Eftimov, G. Ispirova, P. Korošec, and B. Koroušić Seljak, "The RICHFIELDS framework for semantic interoperability of food information across heterogenous information systems", In: FRED, Ana (Ed.), FILIPE, Joaquim (Ed.). Proceedings. Volume 1, KDIR, C3K 2018, 10th International joint Conference on Knowledge Discovery, Knowledge Engineering and Knowledge Management, 18-20 September 2018. [S. 1.]: SCITEPRESS $=$ Science and Technology Publications. 2018, pp. 315-321.

[6] S. Mezgec, T. Eftimov, T. Bucher, and B. Koroušić Seljak, "Mixed deep learning and natural language processing method for fake-food image recognition and standardization to help automated dietary assessment", Public health nutrition, 1-10, 2018.

[7] S. Mezgec and B. Koroušić Seljak, "NutriNet: a deep learning food and drink image recognition system for dietary assessment", Nutrients, ISSN 2072-6643, vol. 9, no. 7, pp. 657-1- 657-19, 2017, doi: 10.3390/nu907065

[8] T. Eftimov, P. Korošec, and B. Koroušić Seljak, "StandFood: standardization of foods using a semi-automatic system for classifying and describing foods according to FoodEx2", Nutrients, 9(6), 542, 2017.

[9] T. Eftimov, B. Koroušić Seljak and P. Korošec, "A rule-based namedentity recognition method for knowledge extraction of evidence-based dietary recommendations", PloS one, 12(6), e0179488, 2017.

[10] B. Koroušić Seljak, P. Korošec, T. Eftimov et al, P. Finglas, "Identification of requirements for computer-supported matching of food consumption data with food composition data", Nutrients, ISSN 2072-6643, 2018, vol. 10, no. 4, str. 433-450, 2018, doi: 10.3390/nu10040433.

[11] M. Kapsokefalou, M. Roe, A. Turrini, H.S. Costa, E. MartinezVictoria, L. Marletta, R. Berry, and P. Finglas. "Food Composition at Present: New Challenges", Nutrients, 2019, vol. 11, 1714; doi:10.3390/nu11081714. 